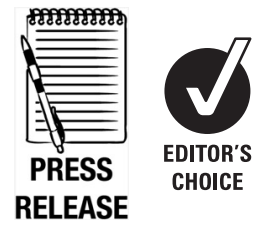

(6) OPEN ACCESS

Department of General Internal Medicine, Division of Psychosomatic Medicine, Inselspital, Bern University Hospital, Bern, Switzerland

\section{Correspondence to}

Professor R von Känel, Department of General Internal Medicine, Division of Psychosomatic Medicine, Inselspital, Bern University Hospital, Freiburgstrasse, $\mathrm{CH}-3010$ Bern, Switzerland; roland.vonkaenel@insel.ch

Accepted 15 October 2010

\title{
Post-traumatic stress in Crohn's disease and its association with disease activity
}

\author{
Rafael J A Cámara, Marie-Louise Gander, Stefan Begré, Roland von Känel; \\ Swiss Inflammatory Bowel Disease Cohort Study Group
}

Objective Violence, accidents and natural disasters are known to cause post-traumatic stress, which is typically accompanied by fear, suffering and impaired quality of life. Similar to chronic diseases, such events preoccupy the patient over longer periods. We hypothesised that post-traumatic stress could also be caused by Crohn's disease (CD), and that CD specific post-traumatic stress could be associated with an increased risk of disease exacerbation.

Methods A cohort of CD patients was observed over 18 months in various types of locations providing gastroenterological treatment in Switzerland. The cohort included 597 consecutively recruited adults. At inclusion, CD specific post-traumatic stress was assessed using the Post-traumatic Diagnostic Scale (range 0-51 points).

During follow-up, clinical aggravation was assessed by combining important outcome measures. Patients with post-traumatic stress levels suggestive of a post-traumatic stress disorder ( $\geq 15$ points) were compared with patients with lower post-traumatic stress levels as well as with patients without posttraumatic stress. Also, the continuous relation between post-traumatic stress severity and risk of disease exacerbation was assessed. Results The $88(19.1 \%)$ patients scoring $\geq 15$ points had 4.3 times higher odds of exacerbation ( $95 \% \mathrm{Cl} 2.6$ to 7.2 ) than the 372 (80.9\%) patients scoring <15 points, and 13.0 times higher odds $(95 \% \mathrm{Cl} 3.6$ to 46.2 ) than the 45 (9.8\%) patients scoring 0 points. The odds of exacerbation increased by 2.2 ( $95 \% \mathrm{Cl} 1.6$ to 2.8 ) per standard deviation of post-traumatic stress.

Conclusions CD specific post-traumatic stress is frequent and seems to be associated with exacerbation of CD. Thus gastroenterologists may want to ask about symptoms of post-traumatic stress and, where relevant, offer appropriate management according to current knowledge.

\section{Introduction}

Crohn's disease (CD) is an incurable inflammatory disease potentially located in the entire gastrointestinal tract with particularly high prevalence rates in Northern Europe, North America and the UK. ${ }^{1}$ The pathological mechanisms are still elusive and the disease course is difficult to predict. The treatment is often exhausting, expensive and burdensome. ${ }^{2}$

Post-traumatic stress is an adverse reaction to traumatic experiences which decreases daily functioning and quality of life and makes the patients suffer. ${ }^{3}$ Patients typically re-experience the traumatic event (eg, in dreams or thoughts), avoid reminders to the event and endure symptoms of hyperarousal (eg, irritability and sleeping difficulties). ${ }^{4}$ Post-traumatic stress is traditionally thought to be caused by violence, accidents and natural disasters. However, research in medical fields other than $\mathrm{CD}$ (eg, cancer, human immunodeficiency virus infection) suggests that diseases (together with the necessary diagnostic and therapeutic procedures) can also be perceived as traumatic events by a considerable number of patients. ${ }^{56}$ To date, CD has not been investigated as a potential cause of post-traumatic stress. This is probably due to the fact that avoidance (such as not talking about the burdensome experience of having CD) is a key feature of post-traumatic stress. However, CD may preoccupy patients over longer periods similar to 'traditional' traumatic events, and we suspect a high prevalence of post-traumatic stress among CD patients.

In addition, post-traumatic stress is associated with lasting alterations in the hypothalamic pituitary adrenal axis, the autonomic nervous system and the immune system, and thus may affect the course of $\mathrm{CD}^{7}$ If caused by myocardial 
infarction, post-traumatic stress is associated with poor cardiovascular prognosis. ${ }^{8}$ Examining the level of post-traumatic stress caused by $\mathrm{CD}$ and its relation to the clinical course of CD seems timely and important given that post-traumatic stress impairs wellbeing and can effectively be treated. ${ }^{9}$

We hypothesised that patients with CD show high levels of post-traumatic stress specific to $\mathrm{CD}$ and, moreover, that $C D$ specific post-traumatic stress is associated with an increased risk of exacerbation of CD over an observation period of 18 months. Because $\geq 15$ points on the Post-traumatic Diagnostic Scale (PDS) is suggestive of a post-traumatic stress disorder (PTSD), our second hypothesis was that patients meeting this cut-off would have a higher risk of adverse events than those scoring below 15 points. ${ }^{10}$

\section{Materials and methods}

\section{Study design and patients}

The ethics committees of all study sites approved the study protocol. Between July 2006 and February 2008, patients were consecutively recruited from virtually all places providing gastroenterological treatment in Switzerland (www.ibdcohort.ch). These included the study centres (ie, University Hospitals of Basel, Bern, Geneva, Lausanne, St Gallen and Zurich), regional hospitals and private practices. Recruitment was performed as follows: the treating gastroenterologists provided their patients with information about the study. When a patient had given written informed consent, gastroenterologists and study nurses completed the enrolment sheet asking about medical history, clinical status and laboratory parameters. More precisely, baseline data included gender, age, disease duration, previous hospitalisations, medication, height, weight, smoking status and the $\mathrm{CD}$ activity index. ${ }^{11}$ At the same time, patients received the PDS with the instruction to complete it and return it directly to the data centre (given that gastroenterologists assessed exacerbation, they had to be blind for post-traumatic stress). To facilitate generalisation of findings to the general population of CD patients treated in Switzerland, the only eligibility criterion was confirmation of a diagnosis of CD based on the criteria of Lennard-Jones ${ }^{12}$; the latter allows confirmation of a diagnosis of $\mathrm{CD}$ by radiological, endoscopic or histological findings, or by surgery.

Subsequent to enrolment and collection of baseline information, patients were followed over an observation period of 18 months. They were advised to inform their treating gastroenterologists whenever they experienced worsening or amelioration of symptoms, in which case an appointment was scheduled to clarify whether fluctuation of symptoms was caused by fluctuation of CD activity or by some other clinical state (eg, infections, gallstones). Regular meetings among gastroenterologists ensured the use of matched diagnostic and therapeutic criteria, as issued by the European Panel on the Appropriateness of CD Treatment. ${ }^{13}$ In addition, the authors informed the data centre in case of inconclusive or missing data in order to improve the enrolment sheet accordingly.

The analysis was performed on an a priori defined sample of 468 patients to assure accurate control for potential confounders without overfitting statistical models. A sample of 367 patients has a power of $90 \%$ to detect an OR of adverse events of 1.5 for an increase of 1 SD in post-traumatic stress at a significance level of $\alpha<0.05$ (two tailed). We computed this power using Power and Precision software for an overall event rate of $25 \%$ over 18 months. ${ }^{14}$ However, we oversampled the calculated sample size by $40 \%$ and enrolled 597 patients (367 corresponds to $60 \%$ of 597) to account for projected loss in response rate, dropouts and missing values.

\section{Measures}

To assess post-traumatic stress we used the German and French version of the PDS, ${ }^{15}$ which is a self-rated questionnaire with 17 items. We replaced the term 'event' by 'intestinal disease' in the PDS questionnaire to ensure measurement of disease specific post-traumatic stress. Each of the three main characteristicsnamely re-experiencing CD ("Did you have bad dreams or nightmares about the intestinal disease?", four items), avoidance of cues related to CD ("Did you try not to think about, talk about or have feelings about the intestinal disease?", seven items) and hyperarousal (five items)-is represented by a separate symptom cluster. All items are rated on a 4 point Likert Scale (0-3; total score 0-51) with a higher score indicating a higher level of post-traumatic stress. Validity measures in our study included a variance of item means of 0.39 and a variance of item variances of 0.01 (ie, good weighting) and a Cronbach's $\alpha$ of 0.84 (ie, good overall reliability). If no more than one item per symptom cluster was missing, replacing it by the mean of the non-missing items of the respective cluster did not affect the validity (personal communication with Stieglitz ${ }^{15}$ in 2009).

Patients scoring $\geq 15$ points are likely to have fully developed PTSD, in which case a structured clinical interview is recommended. ${ }^{4} \mathrm{~A}$ structured clinical interview is mandatory to diagnose full PTSD. Our measure of interest was not PTSD. Instead, we examined post-traumatic stress along a continuum of severity (ie, already at levels far below those seen in fully developed PTSD). This approach has several advantages $^{16}{ }^{17}$ : post-traumatic stress can easily be measured in larger samples by means of the PDS; post-traumatic stress can be detected in patients who may benefit from therapeutic interventions despite not having full PTSD; and ongoing revision of the diagnostic criteria of PTSD cannot affect the validity of the results (http://pn.psychiatryonline.org/content/45/10/8.full; last accessed 14 June 2010). We were primarily interested in the total PDS score but also in re-experience, avoidance and hyperarousal as separate scores. 
Measures of exacerbation of CD were flares, extraintestinal manifestations, complications and non-response to therapy. These were selected because they have a high clinical impact, force patients to seek gastroenterological healthcare and, in combination, allow for a complete evaluation of the disease course. They were combined to one binary outcome which is referred to as adverse events (present/absent), as previously described..$^{18}$ In brief, flares were defined as an increase of 100 points or more from baseline on the $\mathrm{CD}$ activity index. Any need for an increase in medication was considered a treatment failure. Extraintestinal manifestations were included if they occurred during the observation period. Complications included strictures, fistulae, malignancies or any other need for surgery.

Clinical experience has shown that disease duration, strictures and fistulae requiring hospitalisations, need for medication (ie, 5-aminosalicylates, sulfasalazin, steroids, immunosuppressors, antitumour necrosis factor $\alpha$ agents, antibiotics), low body mass index, baseline disease activity and smoking are predictive of the disease course. ${ }^{18}$ For this reason, we controlled not only for those factors but also for gender and age because these are the most important demographic parameters.

\section{Data analysis}

We analysed the data using SPSS 15 for Windows (Chicago, Illinois, USA) excluding the few cases with missing data.

Firstly, we described the total sample. We compared the groups with and without adverse events using standard methods. For mean and percentage differences, we computed asymptotic 95\% CI and two sided $p$ values. We used the $t$ test for normally distributed metric variables (age, body mass index) and the Mann-Whitney $\mathrm{U}$ test for extremely skewed metric variables (diagnosis duration, hospitalisation days related to $C D$ ) or nonmetric quantitative variables (scores). For categorical variables, we compared each category separately using Fisher's exact test.

Secondly, we divided the sample into three groups according to post-traumatic stress levels. Group 1 included all patients with a PDS score of 0 , group 2 included all patients scoring $<15$ (hence this group also included patients in group 1) and group 3 comprised patients scoring $\geq 15$ points. We compared group 3 with groups 1 and 2 in terms of relative risks and ORs of adverse events.

Thirdly, we performed four binary logistic regression models: a model for total post-traumatic stress (primary score), a model for re-experiencing symptoms, a model for avoidance symptoms and a model for hyperarousal symptoms (secondary scores). Each model contained the respective symptom score plus the above mentioned potential confounders as independent variables and adverse events (yes/no) as the outcome.
Fourthly, we performed 16 stratified binary logistic regressions by combing each symptom score with each measure of exacerbation (eg, re-experience and flares) in a different analysis without including potential confounders.

For the combination of total PDS score and adverse events, we set the level of significance at 0.05 and computed 95\% CI for all ORs. For all other combinations, we set the level of significance at 0.01 to adjust for multiple comparisons and computed 99\% CI for the ORs.

\section{Results}

\section{Patient characteristics}

The flowchart (figure 1) shows the selection process of the 468 analysed patients. Sixty-nine patients (14.7\%) experienced flares, $26(5.6 \%)$ did not respond to therapy, $28(6.0 \%)$ suffered from complications and nine (1.9\%) had extraintestinal manifestations. Taking into account the fact that 20 patients experienced more than one category of adverse events (eg, first flares and later on complications), the total number of patients with adverse events was 106 (22.6\%). The characteristics of the whole sample and the differences between the groups with and without adverse events are shown in table 1.

\section{Main results}

Forty-five patients had no post-traumatic stress $(9.8 \%$; group 1), 372 patients scored $<15$ PDS points (80.9\%; group 2 (also includes patients in group 1)) and 88 patients scored $\geq 15$ PDS points (19.1\%; group 3).

Three patients (6.7\%) experienced adverse events in group 1, $64(17.2 \%)$ in group 2 and $42(47.7 \%)$ in group 3 . The odds of experiencing adverse events were thus 0.07 for group 1, 0.21 for group 2 and 0.91 for group 3 . These findings corresponded to an OR of 4.3

We enrolled a total of 597 patients between July 2006 and February 2008

118 patients did not return the questionnaires within 6 months

2 questionnaires were too incomplete for imputation

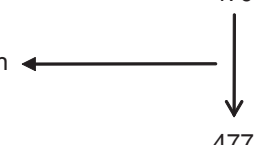

3 patients left Switzerland, 1 patient was untraceable, 5 patients died 4

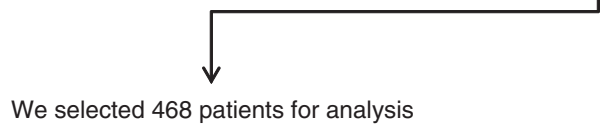

Figure 1 Flowchart of patients selected for analysis, illustrating the number of patients at each stage of the study. Most patients were excluded because they did not return the questionnaires at baseline. We completed 11 re-experience scores, 23 avoidance scores and 15 arousal scores by the mean of the non-missing items. Two Post-traumatic Diagnostic Scales could not be used for analysis because all three subscales (re-experience, avoidance and arousal) had more than one missing value. 
Table 1 Sample characteristics and group differences

\begin{tabular}{|c|c|c|c|c|c|}
\hline Variable & Total $(n=468)$ & Event $+(n=106)$ & Event $-(n=362)$ & Difference $(95 \% \mathrm{Cl})$ & $\mathrm{p}$ Value \\
\hline Total post-traumatic stress & $8.83 \pm 8.08$ & $13.75 \pm 9.22$ & $7.36 \pm 7.08$ & $6.39(4.73 ; 8.05)$ & $<0.001$ \\
\hline Re-experiencing symptoms & $1.84 \pm 2.16$ & $2.86 \pm 2.62$ & $1.54 \pm 1.91$ & $1.32(0.86 ; 1.77)$ & $<0.001$ \\
\hline Avoidance symptoms & $2.91 \pm 3.64$ & $5.17 \pm 4.36$ & $2.24 \pm 3.11$ & $2.93(2.18 ; 3.67)$ & $<0.001$ \\
\hline Hyperarousal symptoms & $4.10 \pm 3.44$ & $5.72 \pm 3.81$ & $3.62 \pm 3.16$ & $2.10(1.38 ; 2.82)$ & $<0.001$ \\
\hline CDAl at baseline & $75.18 \pm 83.14$ & $108.22 \pm 88.16$ & $65.50 \pm 79.16$ & $42.72(23.91 ; 61.52)$ & $<0.001$ \\
\hline Age (years) & $41.97 \pm 14.67$ & $39.70 \pm 15.31$ & $42.64 \pm 14.43$ & $-2.94(-6.11 ; 0.24)$ & 0.041 \\
\hline Female sex & $50.2 \%(235)$ & $58.5 \%(62)$ & $47.8 \%(173)$ & $10.7 \%(-0.1 \% ; 21.5 \%)$ & 0.060 \\
\hline Disease duration (years) & $12.77 \pm 10.38$ & $11.50 \pm 10.55$ & $13.14 \pm 10.32$ & $-1.63(-3.89 ; 0.61)$ & 0.061 \\
\hline Hospitalisation days & $2.24 \pm 7.53$ & $2.87 \pm 6.96$ & $2.05 \pm 7.69$ & $0.82(-0.82 ; 2.45)$ & 0.147 \\
\hline Body mass index & $23.71 \pm 4.62$ & $22.32 \pm 4.39$ & $24.11 \pm 4.62$ & $-1.79(-2.78 ;-0.80)$ & $<0.001$ \\
\hline 5-Aminosalycilates & $16.9 \%(79)$ & $16.0 \%(17)$ & $17.1 \%(62)$ & $-1.1 \%(-9.2 \% ; 7.1 \%)$ & 0.883 \\
\hline Sulfasalazin & $2.1 \%(10)$ & $0.9 \%(1)$ & $2.5 \%(9)$ & $-1.5 \%(-4.7 \% ; 1.6 \%)$ & 0.468 \\
\hline Steroids & $23.3 \%(109)$ & $27.4 \%(29)$ & $22.1 \%(80)$ & $5.3 \%(-3.9 \% ; 14.4 \%)$ & 0.296 \\
\hline Immunosuppressors & $56.6 \%(265)$ & $50.0 \%(53)$ & $58.6 \%(212)$ & $-8.6 \%(-19.3 \% ; 2.2 \%)$ & 0.121 \\
\hline Anti-TNF $\alpha$-agents & $20.3 \%(95)$ & $25.5 \%(27)$ & $18.8 \%(68)$ & $6.7 \%(-2.0 \% ; 15.4 \%)$ & 0.169 \\
\hline Antibiotics & $2.1 \%(10)$ & $2.8 \%(3)$ & $1.9 \%(7)$ & $0.9 \%(-2.2 \% ; 4.0 \%)$ & 0.701 \\
\hline Current smoker & $42.9 \%(200)$ & $48.6 \%(51)$ & $41.3 \%(149)$ & $7.3 \%(-3.5 \% ; 18.1 \%)$ & 0.218 \\
\hline \multicolumn{6}{|l|}{ Education } \\
\hline University degree & $14.5 \%(67)$ & $15.2 \%(16)$ & $14.2 \%(51)$ & $1.0 \%(-6.9 \% ; 8.9 \%)$ & 0.875 \\
\hline Tertiary degree & $15.8 \%(73)$ & $14.3 \%(15)$ & $16.2 \%(58)$ & $-1.9 \%(-9.7 \% ; 5.9 \%)$ & 0.761 \\
\hline Vocational school & $67.8 \%(314)$ & $67.6 \%(71)$ & $67.9 \%(243)$ & $-0.3 \%(-10.5 \% ; 10.0 \%)$ & 1.000 \\
\hline No degree & $1.9 \%(9)$ & $2.9 \%(3)$ & $1.7 \%(6)$ & $1.2 \%(-1.8 \% ; 4.2 \%)$ & 0.430 \\
\hline \multicolumn{6}{|l|}{ Alcohol intake } \\
\hline Daily & $10.1 \%(47)$ & $6.6 \%(7)$ & $11.2 \%(40)$ & $-4.6 \%(-10.4 \% ; 1.2 \%)$ & 0.202 \\
\hline Weekly & $31.7 \%(147)$ & $32.1 \%(34)$ & $31.6 \%(113)$ & $0.5 \%(-9.2 \% ; 10.7 \%)$ & 0.906 \\
\hline$\leq$ Monthly & $58.2 \%(270)$ & $61.3 \%(65)$ & $57.3 \%(205)$ & $4.0 \%(-6.0 \% ; 15.4 \%)$ & 0.502 \\
\hline \multicolumn{6}{|l|}{ Sports activities } \\
\hline Daily & $8.3 \%(37)$ & $8.9 \%(9)$ & $8.1 \%(28)$ & $0.8 \%(-5.5 \% ; 7.2 \%)$ & 0.837 \\
\hline Weekly & $49.3 \%(221)$ & $43.6 \%(44)$ & $51.0 \%(177)$ & $-7.4 \%(-18.6 \% ; 3.7 \%)$ & 0.214 \\
\hline$\leq$ Monthly & $42.4 \%(190)$ & $47.5 \%(48)$ & $40.9 \%(142)$ & $6.6 \%(-4.6 \% ; 17.8 \%)$ & 0.254 \\
\hline \multicolumn{6}{|l|}{ Positive family history } \\
\hline$C D$ & $12.0 \%(56)$ & $17.9 \%(19)$ & $10.2 \%(37)$ & $7.7 \%(0.7 \% ; 14.7 \%)$ & 0.040 \\
\hline Ulcerative colitis/no CD & $2.4 \%(11)$ & $2.8 \%(3)$ & $2.2 \%(8)$ & $0.6 \%(-2.7 \% ; 3.9 \%)$ & 0.718 \\
\hline No inflammatory bowel disease & $85.7 \%(401)$ & $79.2 \%(84)$ & $87.6 \%(317)$ & $-8.3 \%(-15.9 \% ;-0.7 \%)$ & 0.040 \\
\hline
\end{tabular}

Post-traumatic stress: 460 (98.3\%) valid scores; re-experiencing symptoms: 468 (100\%) valid scores; avoidance symptoms: 465 (99.3\%) valid scores; hyperarousal symptoms: $462(98.7 \%)$ valid scores;

CDAl, Crohn's Disease Activity Index at baseline (468 (100\%) valid scores): $20.8 \%$ of patients with and $12.2 \%$ without adverse events had scores between 150 and 300 ( $p=0.038), 1.9 \%$ of patients with and $0.6 \%$ without events had scores between 301 and 450 ( $p=0.222$ ) and none had scores above 450 .

Disease duration, age, days of hospitalisation due to CD, gender, medication and positive family history displayed 468 (100\%) valid indications, body mass index yielded 467 (99.8\%) valid indications, current smoking status showed 466 (99.6\%) valid indications, alcohol intake displayed 464 (99.1\%) valid indications, education had 463 (98.9\%) valid indications and sports activities had 448 (95.7\%) valid indications; $11.3 \%$ of patients with events and $11.9 \%$ without events had no medication $(p=1.000)$.

Qualitative variables are indicated as means \pm SDs and quantitative variables in percentages (with absolute frequencies in parentheses). If no unit is specified, quantitative variables are indicated as score points.

CD, Crohn's disease; TNF, tumour necrosis factor.

(95\% CI 2.6 to $7.2 ; \mathrm{p}<0.001)$ between groups 2 and 3 and 13.0 (95\% CI 3.6 to $46.2 ; \mathrm{p}<0.001)$ between groups 1 and 3 .

After controlling for potential confounders, the odds of experiencing adverse events showed a 2.16fold increase (95\% CI 1.65 to $2.84 ; \mathrm{p}<0.001)$ for an increase of $1 \mathrm{SD}$ of post-traumatic stress, which was the primary measure. In addition, it showed a 1.72fold increase for an increase of $1 \mathrm{SD}$ of re-experiencing symptoms, a 1.77-fold increase for an increase of $1 \mathrm{SD}$ of avoidance symptoms and a 2.07-fold increase for an increase of 1 SD of hyperarousal (table 2). 
Table 2 Results with all predictors

\begin{tabular}{llllll}
\hline & \multicolumn{1}{l}{ Models } & & & \\
\cline { 2 - 6 } Variable & Post-traumatic stress model & Re-experiencing model & Avoidance model & Hyperarousal model \\
\hline TPS & $2.160(1.518 ; 3.093) ;<0.001$ & - & - & - \\
REX & - & $1.721(1.261 ; 2.350) ;<0.001$ & - & - \\
AVO & - & - & $1.771(1.270 ; 2.477) ;<0.001$ & - \\
HYP & - & - & - & $2.070(1.467 ; 2.920) ;<0.001$ \\
CDAl & $1.283(0.920 ; 1.939) ; 0.033$ & $1.514(1.087 ; 2.106) ; 0.004$ & $1.394(0.920 ; 1.939) ; 0.024$ & $1.394(1.017 ; 1.939) ; 0.007$ \\
Female sex B & $1.136(0.586 ; 2.201) ; 0.621$ & $1.357(0.717 ; 2.569) ; 0.217$ & $1.148(0.594 ; 2.216) ; 0.590$ & $1.207(0.632 ; 2.306) ; 0.454$ \\
Age (years) & $1.000(0.972 ; 1.028) ; 0.992$ & $1.000(0.974 ; 1.027) ; 0.994$ & $0.994(0.966 ; 1.022) ; 0.554$ & $1.003(0.977 ; 1.031) ; 0.752$ \\
Disease duration & $0.985(0.948 ; 1.023) ; 0.307$ & $0.988(0.952 ; 1.024) ; 0.380$ & $0.988(0.951 ; 1.026) ; 0.396$ & $0.981(0.946 ; 1.018) ; 0.183$ \\
Hospitalisation days & $0.994(0.957 ; 1.032) ; 0.661$ & $0.994(0.957 ; 1.032) ; 0.676$ & $0.995(0.958 ; 1.034) ; 0.760$ & $0.994(0.958 ; 1.032) ; 0.695$ \\
5-Aminosalycilates B & $0.974(0.400 ; 2.370) ; 0.940$ & $0.920(0.386 ; 2.196) ; 0.805$ & $0.991(0.408 ; 2.411) ; 0.980$ & $0.911(0.381 ; 2.179) ; 0.783$ \\
Sulfasalazin B & $0.025(0.025 ; 7.425) ; 0.447$ & $0.436(0.026 ; 7.329) ; 0.449$ & $0.353(0.021 ; 5.925) ; 0.341$ & $0.429(0.025 ; 7.359) ; 0.443$ \\
Steroids B & $0.813(0.371 ; 1.783) ; 0.497$ & $0.951(0.451 ; 2.004) ; 0.862$ & $0.801(0.366 ; 1.753) ; 0.466$ & $0.859(0.400 ; 1.841) ; 0.607$ \\
Immunosuppressors B & $0.614(0.317 ; 1.191) ; 0.058$ & $0.660(0.347 ; 1.255) ; 0.096$ & $0.637(0.330 ; 1.231) ; 0.078$ & $0.623(0.326 ; 1.188) ; 0.059$ \\
Anti-TNF $\alpha$ B & $1.262(0.565 ; 2.820) ; 0.456$ & $1.340(0.613 ; 2.929) ; 0.335$ & $1.230(0.552 ; 2.738) ; 0.506$ & $1.312(0.599 ; 2.873) ; 0.373$ \\
Antibiotics B & $0.363(0.035 ; 3.737) ; 0.263$ & $0.543(0.070 ; 4.188) ; 0.442$ & $0.398(0.038 ; 4.185) ; 0.313$ & $0.608(0.068 ; 5.441) ; 0.559$ \\
Smoking B & $1.076(0.557 ; 2.077) ; 0.775$ & $1.114(0.588 ; 2.112) ; 0.663$ & $1.092(0.567 ; 2.103) ; 0.730$ & $1.149(0.606 ; 2.181) ; 0.576$ \\
BMI & $0.596(0.395 ; 0.894) ; 0.001$ & $0.624(0.420 ; 0.932) ; 0.002$ & $0.624(0.413 ; 0.937) ; 0.003$ & $0.615(0.411 ; 0.919) ; 0.002$ \\
\hline
\end{tabular}

Values are ORs $(99 \% \mathrm{Cl})$ and corresponding $p$ values.

The OR of disease deterioration for each variable is included. If no unit is specified, quantitative variables indicate ORs for 1 SD. Binary variables (yes/no) are distinguished by a ' $B$ '. Disease duration is indicated in years. Hospitalisation days refers to hospitalisations related to Crohn's disease.

Total Post-traumatic Stress (TPS) Model (457 (97.6\%) valid cases): -2xIn(likelihood)=415; RE-experiencing (REX) Model (465 (99.3\%) valid cases):

$-2 \times \ln ($ likelihood) $=434$; Avoidance (AVO) Model (462 (98.7\%) valid cases): $-2 \times \ln ($ likelihood) $=419$; Hyperarousal (HYP) Model (459 (98.1\%) valid cases):

$-2 \times \ln ($ likelihood) $=431$; Control Model $(465(99.3 \%)$ valid cases): $-2 \times \ln$ (likelihood) $=455 ;-2 \times \ln ($ likelihood ratio PDS/control variables) $=40, p<0.001$;

$-2 \times \operatorname{In}$ (likelihood ratio REX/control variables) $=21, \mathrm{p}<0.001 ;-2 \times \operatorname{In}$ (likelihood ratio AVO/control variables)=36, $\mathrm{p}<0.001 ;-2 \times \ln$ (likelihood ratio ARO/control variables $)=24, p<0.001$.

$\mathrm{BMI}$, body mass index; CDAl, Crohn's Disease Activity Index; TNF, tumour necrosis factor.

The results for the separate disease measures are summarised in table 3 and largely concur with the results for the combined measure.

\section{Discussion}

$\mathrm{CD}$ can be a great burden for patients. $^{2}$ Fatigue, general weakness and hardly foreseeable incidents such as diarrhoea may impair the patient's activities. Treatment is often costly and side effects potentially severe. ${ }^{19}$ Ileostomies, facial bloating and feelings of dirtiness may reduce self-esteem. Severe complications such as perforation and peritonitis may even cause fear of dying. Studies show that patients with CD perceive that their psychological needs are incompletely met. ${ }^{2}$

We found that psychological reactions to CD compare well with reactions to more sudden events, such as violence, accidents and natural disasters. Only $9.8 \%$ of our patients were free of CD specific post-traumatic stress symptoms (group 1) compared with $86.9 \%$ of a sample representing the entire Swiss population (OR 61.6)..$^{20}$ Nineteen per cent of our sample (group 3) scored $\geq 15$ points while only $2 \%$ of the referred sample had subthreshold PTSD (ie, traumatic event with higher levels of post-traumatic stress without the duration and degree of impairment of a full PTSD ${ }^{21}$ ) and none had full PTSD (OR of subthreshold PTSD 11.5).
Distinguishing between subthreshold and full PTSD is important because the therapeutic approaches may be different. Clinical experience has shown that patients with subthreshold PTSD may profit from psychological coaching. Such coaching focuses on improvement of coping capacities, is widely used and results in reduced symptom severity and associated fear. ${ }^{9}$ Full PTSD requires so-called trauma focused therapies to reduce the degree of mental and physical impairment. ${ }^{22}$ In studies on postmyocardial infarction patients, the proportion of patients scoring $\geq 15$ PDS points was the same as in our sample, and in half of those patients clinical PTSD according to DSM-IV criteria was confirmed by a structured interview. ${ }^{23}{ }^{24}$ In anticipation of evidence specific to $\mathrm{CD}$, we recommend treating $\mathrm{CD}$ specific post-traumatic stress in the same way as posttraumatic stress is usually treated.

We also found that the odds of disease exacerbation were four times higher in patients meeting the criterion for a structured clinical interview than in patients scoring below 15 PDS points and even 13 times higher than in patients scoring 0 points. An increase of 1 SD of post-traumatic stress was associated with more than twofold higher odds of subsequent disease deterioration. Isolated symptom clusters of post-traumatic stress showed similarly strong associations. Evidence 


\begin{tabular}{|c|c|c|c|c|}
\hline \multirow[b]{2}{*}{ Outcome } & \multicolumn{4}{|l|}{ Predictors } \\
\hline & Total post-traumatic stress & Re-experiencing symptoms & Avoidance symptoms & Hyperarousal symptoms \\
\hline Flares & $1.626(1.211 ; 2.192) ;<0.001$ & $1.340(0.996 ; 1.801) ; 0.011$ & $1.711(1.279 ; 2.286) ;<0.001$ & $1.477(1.081 ; 2.010) ; 0.001$ \\
\hline Non-response to therapy & $1.689(1.137 ; 2.535) ; 0.001$ & $1.722(1.164 ; 2.541) ;<0.001$ & $1.711(1.174 ; 2.505) ;<0.001$ & $1.366(0.863 ; 2.160) ; 0.079$ \\
\hline Complications & $1.754(1.183 ; 2.590) ;<0.001$ & $1.751(1.197 ; 2.559) ;<0.001$ & $1.668(1.153 ; 2.421) ;<0.001$ & $1.541(1.000 ; 2.369) ; 0.010$ \\
\hline $\begin{array}{l}\text { Extraintestinal } \\
\text { manifestations }\end{array}$ & $1.565(0.829 ; 2.965) ; 0.071$ & $1.443(0.749 ; 2.771) ; 0.150$ & $1.452(0.771 ; 2.740) ; 0.130$ & $1.608(0.796 ; 3.245) ; 0.083$ \\
\hline
\end{tabular}

Values are ORs for 1 SD $(99 \% \mathrm{Cl})$ and corresponding $p$ values.

Sensitivity analyses stratified by the three symptom clusters (re-experiencing, avoidance and hyperarousal) of the Post-traumatic Diagnostic Scale and by the four items of the combined outcome (flares, non-response to therapy, complications and extraintestinal manifestations). For these analyses we did not include additional covariates to avoid overfitted models.

for causality between $\mathrm{CD}$ specific post-traumatic stress and subsequent exacerbation according to the Bradford-Hill criteria included the temporal relationship, strength of the association and pathological findings. ${ }^{25}$ The strength of the association (OR/ SD 2.16 (95\% CI 1.65 to 2.84$)$ ) was similar to that of azathioprine $(1.0-2.5 \mathrm{mg} / \mathrm{kg}$ per day) over placebo in preventing disease exacerbation over 1 year (OR 2.27 (95\% CI 1.75 to 2.94$)) .{ }^{26}$ However, a response relationship between post-traumatic stress and subsequent disease exacerbation has never been examined. A systematic review identified eight studies investigating psychological interventions in CD but none included the treatment of post-traumatic stress. ${ }^{27}$ Post-traumatic stress may contribute to exacerbation by triggering gastrointestinal inflammation. ${ }^{28}$ Elevated levels of proinflammatory biomarkers such as $\mathrm{C}$ reactive protein and tumour necrosis factor $\alpha$, which play an important role in $\mathrm{CD},{ }^{29}$ have been observed in different studies on post-traumatic stress. Given that prior adverse events (or at least their perception as a trauma) predict post-traumatic stress and probably also future adverse events, they could have confounded the association between post-traumatic stress and subsequent adverse events. However, this seems unlikely because we controlled for disease duration, baseline disease activity and prior hospitalisations (a clinically important marker of prior adverse events). In any case, the high prevalence of $\mathrm{CD}$ specific post-traumatic stress remains the more important and more robust finding.

In binary logistic regression an increasing proportion between the number of variables and the number of cases belonging to one of the two possible outcome categories (yes/no) increases the risk of overfitting. We decided to select our number of variables in order to keep an acceptable control of both overfitting and confounding. Cohort studies regularly face the problem of missing values. In the present cohort study, however, the $80.2 \%$ patients who returned the questionnaires lacked a relatively small number of data points (tables 1 and 2). We believe that our sample, being representative of Switzerland, may be representative of other western countries. Interestingly, patients treated in tertiary centres were more likely to participate in the study. We also cannot exclude the possibility that patients with post-traumatic stress were less likely to participate in the study because they avoided being questioned about their disease.

This study is the first to report a point prevalence of CD specific post-traumatic stress, which is high. Clinicians may wish to be aware that a considerable number of patients perceive CD as psychologically traumatic to the extent that the disease preoccupies them in thoughts and dreams and thus causes irritability and sleeping difficulties associated with fear. In most cases patients avoid talking about cues which remind them of having the disease, including intake of medication, which may affect adherence to therapy. ${ }^{2}$ Such behaviour may unwillingly be encouraged by the usual shortness of consultation time and unfamiliarity of clinical gastroenterologists in dealing with the psychological needs of their patients. Nonetheless, it is evident that post-traumatic stress causes considerable suffering. Therefore, gastroenterologists should be aware of the impact of CD on psychological wellbeing and the fact that this relationship is probably bidirectional. This knowledge may open the door to psychological interventions. Gastroenterologists may wish to ask about re-experiencing, avoidance and hyperarousal and, depending on the intensity of symptoms, may be advised to refer patients for psychological counselling or for a structured clinical interview to assess for PTSD. In the case of full PTSD, trauma focused psychotherapy should be considered, which should be performed by a specialist to avoid possible re-traumatisation.

\section{What is already known about this subject}

Patients with Crohn's disease (CD) report that their psychological needs lack attention from the medical community and that genuine information on psychological topics is difficult to obtain.

- Post-traumatic stress, which is traditionally thought to be caused by manmade traumas and natural disasters, is typically accompanied by fear, suffering and reduced quality of life.

- As shown by years of worldwide clinical experience and by high level evidence, the treatment of post-traumatic stress is efficient in reducing fear and suffering. 


\section{What are the new findings}

- Post-traumatic stress is frequently caused by $C D$.

- We observed a strong association between the amount of posttraumatic stress and subsequent disease deterioration.

How might it impact on clinical practice in the foreseeable future?

- The gastroenterologists may want to ask about symptoms of post-traumatic stress and, where relevant, refer the patients for a structured clinical interview in order to search for a posttraumatic stress disorder (PTSD).

- As long as there is no specific evidence for the treatment of post-traumatic stress caused by $C D$, it is reasonable to treat it in the same way as post-traumatic stress caused by other traumatic events.

- If the amount of post-traumatic stress is uncomfortable for the patient but does not reach the duration and impairment of a PTSD, the patient may be offered psychological counselling in order to improve coping with the disease. In the case of a full PTSD, a trauma focused approach by a mental health specialist might be necessary.

- Future studies may examine whether treatment of posttraumatic stress protects from disease deterioration. Although this remains to be proven, other outcome measures (eg, improvement of quality of life, reduction of suffering) are sufficient reasons for treatment.

Contributors RJAC, SB and RvK developed the hypothesis and the study design. RJAC presented the data and drafted the manuscript. RJAC and M-LG analysed and interpreted the findings and performed the literature search. SB and RvK supervised the project. All authors participated in the literature search, revised the manuscript critically and approved the final version.

Acknowledgements The authors acknowledge Annette Kocher for editing assistance and the collaborators of the Swiss Inflammatory Bowel Disease Cohort Study for data collection. In particular, the authors acknowledge the patients for their collaboration.

Funding This work was supported by grant 33CSCO108792 from the Swiss National Science Foundation to RvK and SB (Mental Health Core Project of the Swiss inflammatory Bowel Disease Cohort study). The funding source was not involved in data collection, management, analysis, interpretation or writing, or in the decision to submit the manuscript for publication.

\section{Competing interests None.}

Ethics approval This study was conducted with the approval of the Lausanne University Ethics Committee, Kantonale Ethikkommission Bern, Ethikkommission beider Basel, Ethikkommission des Kantons St Gallen, Kantonale Ethikkommission Zürich and Commission Centrale d'Éthique de la Recherche.

Provenance and peer review Not commissioned; externally peer reviewed.
Open Access This is an Open Access article distributed in accordance with the Creative Commons Attribution Non Commercial (CC BY-NC 3.0) license, which permits others to distribute, remix, adapt, build upon this work noncommercially, and license their derivative works on different terms, provided the original work is properly cited and the use is non-commercial. See: http://creativecommons.org/licenses/by$\mathrm{nc} / 3.0 /$

\section{References}

1. Loftus EV Jr. Clinical epidemiology of inflammatory bowel disease: Incidence, prevalence, and environmental influences. Gastroenterology 2004;126:1504-17.

2. Casati J, Toner BB, de Rooy EC, et al. Concerns of patients with inflammatory bowel disease: a review of emerging themes. Dig Dis Sci 2000;45:26-31.

3. Schnurr PP, Lunney CA, Bovin MJ, et al. Posttraumatic stress disorder and quality of life: extension of findings to veterans of the wars in Iraq and Afghanistan. Clin Psychol Rev 2009;29:727-35.

4. Diagnostic and Statistical Manual of Mental Disorders, 4th Edn. Arlington, VA: American Psychiatric Association 2004.

5. Gurevich M, Devins GM, Rodin GM. Stress response syndromes and cancer: conceptual and assessment issues. Psychosomatics 2002;43:259-81.

6. Tedstone JE, Tarrier N. Posttraumatic stress disorder following medical illness and treatment. Clin Psychol Rev 2003;23: 409-48.

7. Heim C, Nemeroff CB. Neurobiology of posttraumatic stress disorder. CNS Spectr 2009;14(Suppl 1):13-24.

8. Shemesh E, Yehuda R, Milo O, et al. Posttraumatic stress, nonadherence, and adverse outcome in survivors of a myocardial infarction. Psychosom Med 2004;66:521-6.

9. Bisson J, Andrew M. Psychological treatment of posttraumatic stress disorder (PTSD). Cochrane Database Syst Rev 2005;18:CD003388.

10. Foa EB, Cashman L, Jaycox L, et al. The validation of a self-report measure of posttraumatic stress disorder: the posttraumatic diagnostic scale. Psychol Assess 1997;9:445-51.

11. Best WR, Becktel JM, Singleton JW, et al. Development of a Crohn's disease activity index. National Cooperative Crohn's Disease Study. Gastroenterology 1976;70:439-44.

12. Lennard-Jones JE. Classification of inflammatory bowel disease. Scand J Gastroenterol Suppl 1989;170:2-6.

13. Michetti P, Stelle M, Juillerat P, et al. Appropriateness of therapy for active Crohn's disease: results of a multidisciplinary international expert panel-EPACT II. J Crohn Colitis 2009;3:232-40.

14. Borenstein M, Rothstein H, Cohen J. Power and precision. Englewood, NJ: Biostat Inc. 2001.

15. Stieglitz RD, Frommberger U, Foa EB, et al. Evaluation of the German version of the PTSD Symptom Scale (PSS). Psychopathology 2001;34:128-33.

16. Mol SS, Arntz A, Metsemakers JF, et al. Symptoms of post-traumatic stress disorder after non-traumatic events: evidence from an open population study. Br J Psychiatry 2005;186:494-9.

17. Rosen GM, Spitzer RL, McHugh PR. Problems with the posttraumatic stress disorder diagnosis and its future in DSM V. Br J Psychiatry 2008;192:3-4.

18. Cámara RJ, Lukas PS, Begré S, et al; Swiss Inflammatory Bowel Disease Cohort Study (SIBDCS) group. Effects of social support on the clinical course of Crohn's disease. Inflamm Bowel Dis 2010 Oct 25. [Epub ahead of print]. 
19. Baumgart DC, Sandborn WJ. Inflammatory bowel disease: clinical aspects and established and evolving therapies. Lancet 2007;369:1641-57.

20. Hepp U, Gamma A, Milos G, et al. Prevalence of exposure to potentially traumatic events and PTSD. The Zurich Cohort Study. Eur Arch Psychiatry Clin Neurosci 2006;256:151-8.

21. Stein MB, Walker JR, Hazen AL, et al. Full and partial posttraumatic stress disorder: findings from a community survey. Am J Psychiatry 1997;154:1114-19.

22. Cloitre M. Effective psychotherapies for posttraumatic stress disorder: a review and critique. CNS Spectr 2009;14(Suppl 1):32-43.

23. Wiedemar L, Schmid JP, Müller J, et al. Prevalence and predictors of posttraumatic stress disorder in patients with acute myocardial infarction. Heart Lung 2008;37:113-21.

24. Guler E, Schmid JP, Wiedemar L, et al. Clinical diagnosis of posttraumatic stress disorder after myocardial infarction. Clin Cardiol 2009;32:125-9.
25. Hill AB. The environment and disease: association or causation? Proc R Soc Med 1965;58:295-300.

26. Pearson DC, May GR, Fick GH, et al. Azathioprine and 6-mercaptopurine in Crohn disease. A meta-analysis. Ann Intern Med 1995;123:132-42.

27. Cámara RJ, Begré S, von Känel R. The effect of stressrelieving interventions on inflammatory bowel disease: quality assessment of 10 therapeutic studies. Z Psychosom Med Psychother 2010;56:116-35.

28. von Känel R, Begré S, Abbas CC, et al. Inflammatory biomarkers in patients with posttraumatic stress disorder caused by myocardial infarction and the role of depressive symptoms. Neuroimmunomodulation 2010;17:39-46.

29. Vermeire S, Van Assche G, Rutgeerts P. C-reactive protein as a marker for inflammatory bowel disease. Inflamm Bowel Dis 2004;10:661-5. 